\title{
AUTOPSIE-STUDIE
}

\section{Schützen Sartane vor Alzheimer?}

Bei Patienten, die mit Angiotensin-Rezeptorblockern behandelt werden, finden sich autoptisch seltener pathologische Befunde, die für die Alzheimerkrankheit typisch sind. 890 hypertone Patienten (Durchschnittsalter 81 Jahre), die zuvor Hinweise auf kognitive oder hirnpathologische Beeinträchtigungen gezeigt hatten, wurden autopsiert. 133 von ihnen waren mit Sartanen, 577 mit anderen Antihypertensiva und 180 nicht medikamentös behandelt worden. Die
Wahrscheinlichkeit, dass die pathologische Untersuchung des Gehirns eine Alzheimerdiagnose nach sich zog, war bei den mit Sartanen behandelten Patienten rund 35\% niedriger als bei jenen, die ein anderes Antihypertensivum erhalten hatten. Dasselbe Resultat zeigte der Vergleich mit den unbehandelten Hypertonikern. Die ermittelten Unterschiede waren statistisch relevant.

Arch Neurol. Published online September 10, 2012; doi:10.1001/archneurol.2012.1010

\section{ÜBERLASTET UND UNZUFRIEDEN}

\section{Dann macht die Arbeit Kopfschmerzen}

Vielen Berufstätigen machen Kopfschmerzen zu schaffen. Das liegt nicht selten auch an den Arbeitsbedingungen. Besonders gefährdet sind Angestellte, die ein hohes Arbeitspensum bewältigen müssen oder ihre Tätigkeit als unbefriedigend empfinden, ergab eine prospektive Studie aus Norwegen. 3574 Angestellte, von ungelernten Verkäufern bis zu Managern, hatten im Abstand von zwei Jahren zu Arbeitsbedingungen und Kopfschmerzen Auskunft gegeben. Es gibt wahrscheinlich mehrere
Wege, auf denen eine belastende Arbeitssituation zu Kopfschmerzen führen kann: Zum Beispiel kann sie bestimmte neurale und endokrine Signalwege aktivieren oder die Toleranz gegenüber anderen expositionellen "Noxen" herabsetzen. Ängste scheinen außerdem die Signalweiterleitung in absteigenden schmerzhemmenden Bahnen zu unterdrücken.

Pain 2012; Online first 18 August 2012. http://dx.doi. org/10.1016/j.pain.2012.07.009

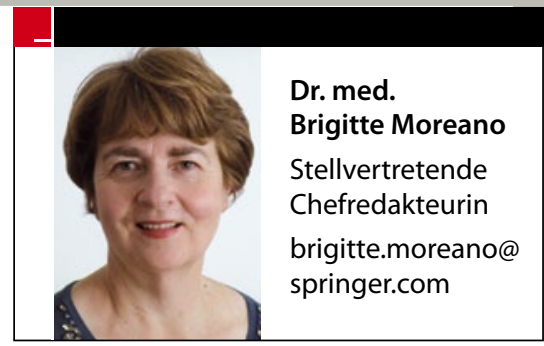

ALKOHOLISMUS

\section{Saufen, bis das Hirn blutet}

Wer zu reichlichem Alkoholkonsum neigt, hat ein höheres Risiko, schon in jüngeren Jahren eine intrazerebrale Blutung zu erleiden und daran zu versterben. Dies ergab eine französische Studie an Patienten mit Hirnblutung. 25\% dieser Patienten mit einer Hirnblutung fielen durch problematischen Alkoholkonsum auf. Wer regelmäßig viel trinkt, muss 14 Jahre früher mit einer Hirnblutung rechnen als einer, der sich aus Alkohol nicht so viel macht. Neurology 2012;79:1109-1115

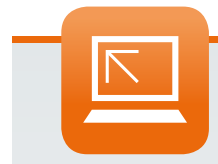

www.springermedizin.de/webtv

WebTV von springermedizin. de bietet Ihnen aktuelle VideoInterviews mit Experten aller Fachrichtungen. Im September stehen die Videos von der Jahrestagung der European Society of Cardiology im Fokus:

Die neuen Leitlinien zur Herzinsuffizienz 3214496 Neue Aspekte der Hypertoniebehandlung 3214466 Antikoagulationstherapie bei akutem Koronarsyndrom 3215422

Die Beiträge finden Sie, indem Sie die ID-Nummer in die Suche eingeben, das WebTV über den nebenstehenden QR-Code. einen der genannten Parameter untersucht hatten. Die Ergebnisse rechtfertigen weder eine Behandlung mit Omega-3-FettsäureKapseln noch Empfehlungen zur diätetischen Zufuhr. Die europäische Kardiologengesellschaft (ESC) rät in ihrer neuen Leitlinie zum Herzinfarkt nicht mehr zur Einnahme von Omega-3-Fettsäuren. Bestand hat dagegen die Empfehlung, zur KHK-Prävention regelmäßig Fisch zu essen.

JAMA 2012;308(10):1024-1033 domisierten Studien herangezogen worden, die mindestens ein Jahr gedauert und 